\title{
Paraneoplastic Hyperleukocytosis in Pancreatic Adenocarcinoma
}

\author{
Evan S. $\mathrm{Wu}^{1, *}$ and Samer A. Srour ${ }^{2}$
}

\begin{abstract}
Background: Paraneoplastic hyperleukocytosis is a rare phenomenon observed in patients with adenocarcinomas and other malignancies. In this study, we present a case of paraneoplastic hyperleukocytosis in pancreatic adenocarcinoma secondary to excessive secretion of serum granulocyte colony-stimulating factor (G-CSF).

Presentation: We report a 71-year-old Caucasian male who presented to our hospital with hyperleukocytosis in the setting of locally advanced pancreatic adenocarcinoma. The patient was recently diagnosed 4 months before presentation and received abraxane/gemcitabine in addition to palliative radiation therapy for continued gastrointestinal bleeding. During routine laboratory assessment, the patient was found to have a white blood cell (WBC) count of $153 \mathrm{~K} / \mathrm{UL}$ (75\% neutrophils and 14\% bands). Bone marrow biopsy and cytology were negative for neoplastic features. Serum G-CSF levels returned markedly elevated, supporting the diagnosis of paraneoplastic hyperleukocytosis. Interestingly, the WBC count decreased significantly following each of two cycles of chemotherapy, further suggesting a paraneoplastic etiology of hyperleukocytosis. The patient did not receive any growth factor support at any point before or during treatment.

Conclusion: In conclusion, the presence of hyperleukocytosis in cancer should raise clinical suspicion of a paraneoplastic phenomenon when other possible causes have been excluded. Hyperleukocytosis in this setting may correlate with progression of disease and lessen with treatment.
\end{abstract}

Keywords: hyperleukocytosis; paraneoplastic syndromes; pancreatic adenocarcinoma

\section{Background}

Paraneoplastic syndromes are clinical disorders associated with malignancy, which are not directly related to the physical effects of the primary or metastatic tumor. ${ }^{1,2}$ Hyperleukocytosis refers to a laboratory abnormality that has been variably defined as total leukocyte count greater than 50 or $100 \mathrm{~K} / \mathrm{UL}$. Hyperleukocytosis is a rare paraneoplastic syndrome that has been observed in association with renal, pancreatic, and lung cancers. ${ }^{3,4}$ This report describes a case of hyperleukocytosis that developed in a patient with locally advanced pancreatic adenocarcinoma.

\section{Case Presentation}

A 71-year-old male presented to our hospital with symptomatic anemia and hyperleukocytosis. The patient was recently diagnosed 4 months before with locally advanced pancreatic adenocarcinoma given that there was no evidence of metastatic disease and the tumor was determined to be unresectable based on the extent of local vascular involvement. His past medical history was only significant for hypertension, and social history was negative for tobacco or alcohol use. Family history was negative for cancer in his immediate family and review of symptoms was unremarkable,

${ }^{1}$ Department of Internal Medicine, Baylor College of Medicine, Houston, Texas.

${ }^{2}$ Division of Hematology and Oncology, Baylor College of Medicine, Houston, Texas.

*Address correspondence to: Evan S. Wu, MD, Department of Internal Medicine, Baylor College of Medicine, 1463 Drew Street, Houston, TX 77004, E-mail: nave119@gmail.com

(C) Evan S. Wu and Samer A. Srour 2017; Published by Mary Ann Liebert, Inc. This is an Open Access article distributed under the terms of the Creative Commons Attribution License, which permits unrestricted use, distribution, and reproduction in any medium, provided the original work is properly cited. 


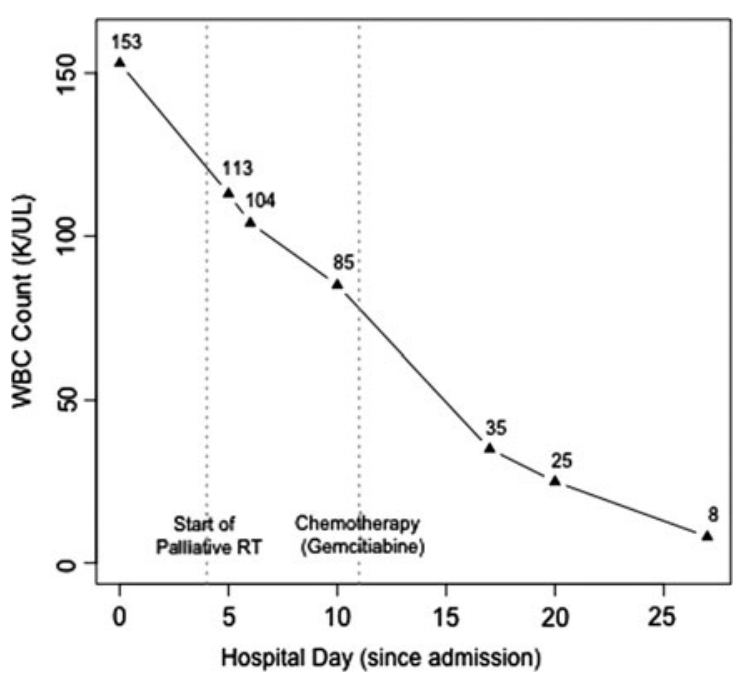

FIG. 1. White blood cell count (K/UL) by hospital day.

apart from general fatigue and decreased activity. On physical examination, the patient was afebrile, his blood pressure and heart rate were normal, no lymphadenopathy or abdominal tenderness was noted, and lung sounds were clear. The laboratory data (complete blood count, chemistry, urinalysis, tumor markers, and coagulation) showed severe anemia $(6.7 \mathrm{~g} / \mathrm{dL}$ of hemoglobin) and thrombocytosis $(625 \mathrm{~K} / \mathrm{UL})$, and the tumor marker carbohydrate antigen 19-9 (CA 19-9) was within the reference range at $22 \mathrm{U} / \mathrm{mL}$. The lactate dehydrogenase (LDH) and alkaline phosphatase (ALP) were elevated to 392 and $493 \mathrm{IU} / \mathrm{L}$, respectively. Marked hyperleukocytosis, $153 \mathrm{~K} / \mathrm{UL}$, was noted with $75 \%$ neutrophils, $14 \%$ bands, and $8 \%$ monocytes. We proceeded to workup the hyperleukocytosis with additional testing. Our differential included paraneoplastic etiology, bone marrow invasion, infection, and hematologic malignancy (e.g., chronic neutrophilic leukemia). Bone marrow biopsy showed a hypercellular (95\%) marrow with trilineage hematopoiesis, myeloid predominance, and $1 \%$ blasts. Pankeratin immunostaining did not identify metastatic carcinoma in the bone marrow. Granulocytes comprised the majority of the myeloid population with monocytes representing 3\% of total cells. Cytogenetic investigation showed a normal chromosome set $(46, \mathrm{XY})$ and hematologic malignancy panel was negative, including JAK2, CSF1R and BCR-ABL. Serum granulocyte colony-stimulating factor (G-CSF) level was significantly elevated at $311 \mathrm{pg} / \mathrm{mL}$ (reference range <39.1).
Based on these findings, the patient was diagnosed with likely paraneoplastic hyperleukocytosis secondary to excessive endogenous secretion of G-CSF. This diagnosis was further supported by the temporal relationship between treatment with abraxane/gemcitabine and palliative radiation therapy, and the decrease in white blood cell count (Fig. 1). It is important to note that the patient did not receive any growth factor support at any point before or during treatment. Unfortunately, after one cycle of chemotherapy, reimaging revealed that the patient's tumor had spread to the lungs and the liver and the patient and his family opted for home hospice rather than further treatment.

\section{Conclusions}

In a patient with pancreatic adenocarcinoma, leukocytosis can be due to infection, use of corticosteroid or hematopoietic growth factors, bone marrow involvement, new hematologic malignancy, or paraneoplastic manifestation. Paraneoplastic hyperleukocytosis is a rare phenomenon that is generally associated with a poor prognosis and aggressive disease. ${ }^{5,6}$ There have been eight published cases of granulocyte-colony stimulating factor-producing pancreatic cancer with an associated leukocytosis ranging from 14 to $46 \mathrm{~K} / \mathrm{UL}$. One of the cases reported by Joshita et al. (2009) defined this phenomenon of paraneoplastic hyperleukocytosis with the following diagnostic criteria: (1) extreme leukocytosis (>100 K/UL), (2) elevated G-CSF activity, and (3) falloff of white blood cell count after tumor resection. ${ }^{7}$ While the mechanism for the aggressive nature of these particular types of tumors is not fully understood, it has been shown in vivo that G-CSF stimulates the growth of nonhematopoietic malignant cell lines ${ }^{8,9}$ and is also considered to be a major autocrine growth factor in rapid tumor proliferation and metastasis. ${ }^{10,11}$ In conclusion, the presence of hyperleukocytosis in patients with advanced malignancy should raise clinical suspicion of a paraneoplastic phenomenon, particularly in patients without an infective focus. Moreover, hyperleukocytosis in this setting may suggest an exceptionally aggressive course of disease.

\section{Author Disclosure Statement}

No competing financial interests exist.

\section{References}

1. Kanaji N, Watanabe N, Kita N, et al. Paraneoplastic syndromes associated with lung cancer. World J Clin Oncol. 2014;5:197-223.

2. Farolfi A, Ferrario C, Aquilina M, et al. Paraneoplastic hypocalcemiainduced heart failure in advanced breast cancer: a case report and literature review. Oncol Lett. 2015;10:773-777. 
3. Sreevatsa A, Babu SM, Babu GK, et al. Hyperleukocytosis, an unusual paraneoplastic manifestation of lung cancer: case report and review of literature. J Cancer Res Ther. 2015;11:669.

4. Van Rossum AP, Vlasveld LT, Vlasveld IN, et al. Granulocytosis and thrombocytosis in renal cell carcinoma: a pro-inflammatory cytokine response originating in the tumour. Neth J Med. 2009;67:191-194.

5. Negrier S, Escudier B, Gomez F, et al. Prognostic factors of survival and rapid progression in 782 patients with metastatic renal carcinomas treated by cytokines: a report from the Groupe Francais d'Immunotherapie. Ann Oncol. 2002;13:1460-1468.

6. Guida M, Casamassima A, Monticelli G, et al. Basal cytokins profile in metastatic renal cell carcinoma patients treated with subcutaneous IL-2 based therapy compared with that of healthy donors. J Translational Med. 2007;5:51-57.

7. Joshita S, Nakazawa K, Sugiyama Y, et al. Granulocyte-colony stimulating factor-producing pancreatic adenosquamous carcinoma showing aggressive clinical course. Inter Med. 2009;48:687-691.

8. Segawa K, Ueno $Y$, Kataoka T. In vivo tumor growth enhancement by granulocyte colony-stimulating factor. Jpn J Cancer Res. 1991;82:440447.

9. Asano $\mathrm{S}$, Urabe A, Okabe T, et al. Demonstration of granulopoietic factor(s) in the plasma of nude mice transplanted with a human lung cancer and in the tumor tissue. Blood. 1977;49:845-852.
10. Tachibana M, Miyakawa A, Tazaki H, et al. Autocrine growth of transitional cell carcinoma of the bladder induced by granulocyte colony stimulating factor. Cancer Res. 1995;55:3438-3443.

11. Baba $M$, Hasegawa $H$, Nakayabu $M$, et al. Establishment and characteristics of a gastric cancer cell line (HuGC-OOHIRA) producing high levels of G-CSF, GM-CSF, and IL-6: the presence of autocrine growth control by GCSF. Am J Hematol. 1995;49:207-215.

Cite this article as: Wu ES, Srour SA (2017) Paraneoplastic hyperleukocytosis in pancreatic adenocarcinoma, Journal of Pancreatic Cancer 3:1, 84-86, DOI: 10.1089/pancan.2017.0019.

\section{Abbreviations Used}

$\mathrm{ALP}=$ alkaline phosphatase

$\mathrm{G}-\mathrm{CSF}=$ granulocyte colony-stimulating factor $\mathrm{LDH}=$ lactate dehydrogenase

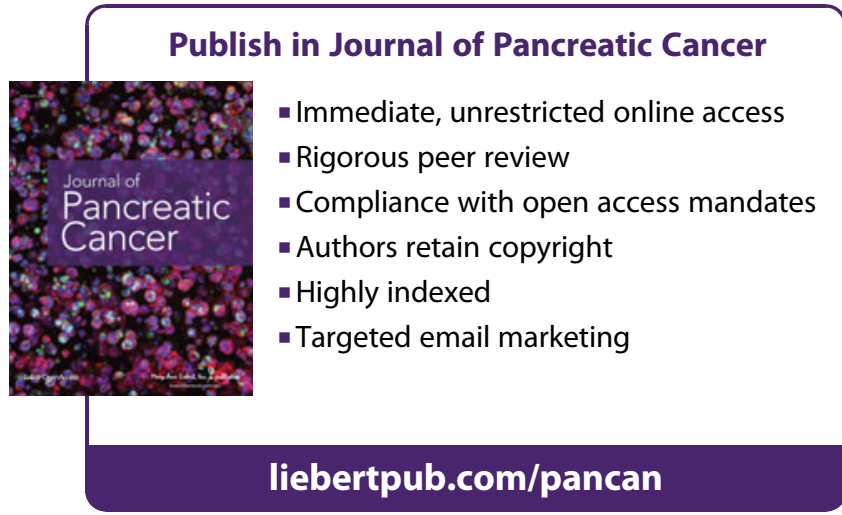

